\title{
Segregation Effects in Polyethylene Blends Revealed by Transmission Electron Microscopy
}

\author{
Cristián C. Puig
}

Grupo de Polímeros USB, Departamento de Ciencias de los Materiales, Universidad Simón Bolívar, Apartado 89000, Caracas 1080-A, Venezuela.

It is usual to find that regular and flexible polymer chains crystallize at large extent on cooling from the melt. The final crystallinity depends on the thermal treatment imposed upon the polymer. The properties of these polymeric materials are affected by the presence of crystals and their perfection. Polyethylene (PE) is a semicrystalline polymer that finds multiple uses in the plastic industry. The presence of branches along the macromolecular chains, disrupting their regularity, causes a lowering in crystallinity and melting temperature. Polyethylene microstructure may be examined by transmission electron microscopy (TEM) and other techniques such as differential scanning calorimetry (DSC) and small angle X-ray scattering (SAXS).

A common method used to study polymer morphology by TEM is to stain it with a strong agent and then producing thin sections that are finally observed under the electron beam. For polyethylene, chlorosulphonic acid serves as staining agent, producing black colored samples, being indicative that surface oxidation had taken place [1]. Morphology studies show PE crystals are usually thin and their lateral dimensions (at right angles to the chain direction) are relatively longer. Their exact dimensions are dependent on the crystallization conditions and chemical structure.

Polyethylenes of various types are usually mixed in order to improve processability and the toughness of the final product. In this work, we examined blends of linear and branched polyethylene, and chlorosulphonic acid was used as staining agent. Staining and the sectioning process using a Leica ultramicrotome were carried out at room temperature. Sections were soaked in uranyl acetate. A transmission electron microscope Jeol JEM-1220, operated at 100kV, was used to reveal the lamellar morphology.

Figure 1 shows the lamellar morphology of a 1\% Linear polyethylene (LPE) / 99\% Branched polyethylene (BPE) blend. Isothermal thermal treatments were carried out in order to segregate both components in the blend. Three lamellar populations are distinguishable, one formed through crystallization at higher temperatures $\left(122^{\circ} \mathrm{C}\right)$, composed of long and thicker LPE lamellae. The presence of branches in BPE impedes its crystallization at such high temperatures, remaining in the melt over the entire isothermal thermal treatment, as previously described by Norton and Keller [2]. Mostly, these chains crystallize later on cooling resulting in short and thin lamellae. In addition, a third population of lamellae formed at $114^{\circ} \mathrm{C}$ may be observed in Figure 1. This is composed of lamellae that are long but thinner than those lamellae formed at $122^{\circ} \mathrm{C}$. A characteristic feature of these lamellae is that they branch out from the thicker lamellae. Complete crystallization of LPE occurred at $122^{\circ} \mathrm{C}$, therefore, these crystals formed at $114^{\circ} \mathrm{C}$ are composed of BPE molecules that nucleate at the surface of the preexisting LPE crystals favoring their growth.

\section{References}


[1] G. Kanig, Colloid Polym Sci., 255 (1977) 1005.

[2] D. Norton, A. Keller, J. Mat. Sci. 19 (1984) 447.

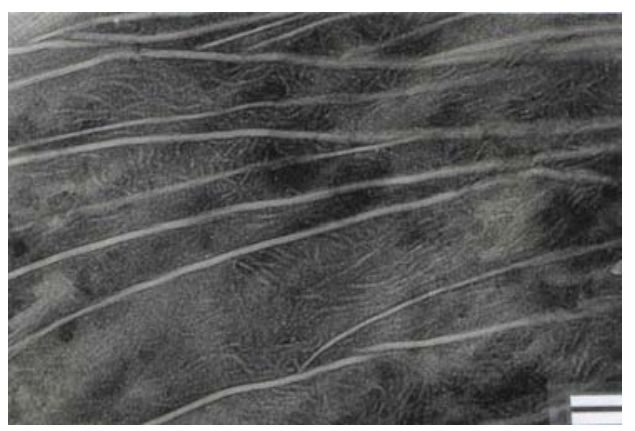

FIG. 1. Electron micrograph showing a section of 1\%LPE blend after chlorosulphonation. Microscale represents $0.1 \mu \mathrm{m}$. 